\title{
Antipsychotic drugs increase Neuregulin1b1 serum levels in first-episode drug-naïve patients and chronic schizophrenia with suggestions for improving the treatment of psychotic symptoms
}

\section{Haidong Yang}

The Fourth People's Hospital of Lianyungang, The Affiliated KangDa College of Nanjing Medical

University

\section{Wen Pan}

Institute of Mental Health, The Affiliated Guangji Hospital of Soochow University

\section{Wenhuan Xiao}

Affiliated WuTaiShan Hospital of Medical College of Yangzhou University

\section{Man Yang}

The Fourth People's Hospital of Lianyungang, The Affiliated KangDa College of Nanjing Medical University

\section{Jianchun Xu}

The Fourth People's Hospital of Lianyungang, The Affiliated KangDa College of Nanjing Medical University

Jin Li

Institute of Mental Health, The Affiliated Guangji Hospital of Soochow University

Xiaobin Zhang ( $\square$ zhangxiaobim@163.com)

Institute of Mental Health, The Affiliated Guangji Hospital of Soochow University

\section{Research Article}

Keywords: antipsychotic drugs, Neuregulin1, FEDN, schizophrenia

Posted Date: September 23rd, 2021

DOI: https://doi.org/10.21203/rs.3.rs-929283/v1

License: (a) This work is licensed under a Creative Commons Attribution 4.0 International License. Read Full License 


\section{Abstract}

Background: Neuregulin1 (NRG1) plays a role in neuronal migration, regulation of synaptic plasticity, and neural survival, and has been considered to be among the candidate genes for schizophrenia. This study focused on the variations in serum NRG1b1 levels following antipsychotic treatment and the relationship between NRG1b1 level and improvements in psychotic symptoms in first-episode drug-naïve (FEDN) patients and chronic schizophrenia.

Methods: A total of 100 patients with schizophrenia were recruited and compared with 79 matched healthy controls. All patients had been drug-naïve for at least four weeks. Serum NRG1b1 levels and positive and negative syndrome scale (PANSS) scores were measured the baseline and after four weeks. Serum NRG1b1 levels were measured using sandwich enzyme-linked immunosorbent assays (ELISA).

Results: Baseline NRG1b1 levels were significantly lower in the patients with schizophrenia compared with the healthy controls. NRG1b1 levels increased significantly following antipsychotic treatment. NRG1b1 levels gradually increased with declining PANSS scores and its three subscales during antipsychotic therapy. The levels of NRG1b1 increased significantly in responders after four weeks of treatment, although non-responders showed no such effect. Correlation analyses showed that the levels of NRG1b1 were negatively correlated with the duration of illness and positively correlated with improvement in symptoms.

Conclusion: The levels of serum NRG1b1 and the therapeutic effects gradually increased following treatment, indicating that NRG1b1 may be an indicator of therapy, and that it may also be associated with the pathophysiological mechanism causing schizophrenia, although this possible pathway requires further investigation. Antipsychotic drugs increase Neuregulin1b1 serum levels in first-episode drug-naïve patients and chronic schizophrenia with suggestions for improving the treatment of psychotic symptoms

\section{Introduction}

Schizophrenia is one of the most severe and complex mental disorders and affects nearly $1 \%$ of the global population; its rising prevalence contributes a greater burden in underdeveloped countries [1]. Nevertheless, only approximately half of patients with schizophrenia receive satisfactory treatment because its symptoms vary and its underlying pathogenetic mechanisms and antipsychotic treatments, and their side effects are poorly understood [2]. The evidence suggests that neurotrophic factors (NTFs) play a crucial role in the pathogenesis of schizophrenia, and neurotrophic hypotheses have gained momentum with the development of antipsychotic therapies $[3,4]$.

Neuregulin (NRG) is an NTF closely related to the development of the central nervous system [5] and comprises NRG1-4 isoforms, among which NRG1, and its receptors ErbB4, are thought to be among the susceptibility genes for schizophrenia. Moreover, alterations to the NRG1 and ErbB4 signaling pathway are involved in neuronal migration, regulation of synaptic plasticity and neural survival, which may be the 
neurobiological underpinnings of schizophrenia [6, 7]. Most importantly, more and more studies have reported that NRG1-ErbB4 signaling is related to glutamatergic, GABAergic and dopaminergic neurotransmission [8] and plays a vital role in the psychopathological mechanism of schizophrenia, and may be useful in evaluating the effect of antipsychotic medication therapies or new drug developments [9]. Subsequently, evidence from studies of mouse models have demonstrated that overexpression of heterozygous NRG1 type III in mice regulates schizophrenia-relevant behaviors, indicating that NRG1 is associated with abnormal behavioral phenotypes in schizophrenia [10]. In addition, a systematic review has implied that antipsychotic drugs can modify some abnormal behaviors in NRG1 and ErbB4 knockout transgenic mouse models, and can affect the expression of NRG1/ ErbB4 and the functioning of NRG1ErbB4 signaling pathways [11].

A post-mortem study reported that NRG1 type $\otimes$ mRNA expression in hippocampal tissue increased in schizophrenic patients compared with controls [12]. In contrast, Parlapani et al. found that the expression

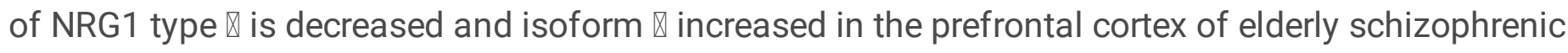
patients, indicating that the altered expression of NRG1 may play an important role during neurodevelopment, neuronal migration and differentiation in schizophrenia [13]. In addition, an imaging genetic study suggested that the risk T allele of SNP8NRG243177, which has been identified as a functional single-nucleotide polymorphism (SNP) in a regulatory domain of NRG1 that impacts on NRG1 function, can contribute to the enlargement of the lateral ventricles observed in early phases of schizophrenia [14]. Genetic variation in NRG1 has also been associated with hippocampal volume reductions in Icelandic schizophrenia patients and in non-affected family members [15].

A previous study found that NRG1 mRNA expression in peripheral lymphocytes is abnormally low in first onset schizophrenia, and that this expression gradually increases in antipsychotic treated patients, demonstrating that NRG1 mRNA may serve as a potential therapeutic marker [16]. Another study in an Australian cohort showed that serum NRG1 11 protein levels are lower in clozapine-treated schizophrenia patients compared with healthy controls, while there is no difference in the mRNA expression of NRG1 type $\otimes$ [17]. Research by Kastin indicated that NRG-1 can enter the spinal cord and brain by a saturable receptor-mediated mechanism, indicating the possibility that NRG-1-b1 may be a promising candidate in central nervous system therapeutics [18].

Taken together, these studies have provided evidence that abnormal expression and function of NRG1 may be associated with pathophysiological mechanisms and therapeutic effects in schizophrenia, although these studies have failed to show a consistent relationship between NRG1 and schizophrenia. Accordingly, we hypothesized that antipsychotic treatment may alter NRG1 levels. This study was designed to determine: (1) whether serum NRG1 $\beta 1$ levels were altered after atypical antipsychotic treatment; and (2) whether alterations in serum NRG1 131 level were correlated with improvements in psychotic symptoms in FEDN patients and chronic schizophrenia. Previously, only a few studies have explored the relationship between serum NRG1 $\beta 1$ levels and the therapeutic effect of antipsychotic treatment in schizophrenic patients of Han Chinese ancestry. 


\section{Methods}

\subsection{Subjects, assessment and study procedures}

A total of 69 (male/female $=34 / 35)$ FEDN patients and 31 (male/female $=21 / 10)$ chronic schizophrenia patients were recruited from the Wu Tai Shan Hospital, YangZhou, China. The chronic patients were drugnaïve for at least four weeks. The total male/female ratio was $55 / 45$, the mean age ( \pm standard deviation SD) was $35.07 \pm 11.42$ years, the mean period in education was $10.40 \pm 3.43$ years, the mean age at schizophrenia onset was $29.59 \pm 10.64$ years, and the mean duration of illness was $5.36 \pm 7.58$ years. All patients were confirmed as schizophrenic using the Structured Clinical Interview of the Diagnostic and Statistical Manual-IV (SCID). Psychotic symptoms were assessed by two experienced psychiatric specialists using positive and negative syndrome scale (PANSS) score before treatment and four weeks after treatment. The inter-rater correlation coefficient for the PANSS score was $>0.8$. The patient exclusion criteria were epilepsy, mental retardation, dementia, traumatic or chronic brain injury, alcohol or substance dependence/abuse, thyroid diseases, diabetic peripheral neuropathy, and patients currently under psychopharmacological treatment.

Patients were treated with atypical antipsychotic drugs, including: clozapine $(n=7)$, risperidone $(n=16)$, ziprasidone $(n=5)$, quetiapine $(n=8)$, olanzapine $(n=6)$, amisulpride $(n=13)$, aripiprazole $(n=8)$, or a combination of these antipsychotics $(n=37)$. Antipsychotic dosages were personalized according to the clinical symptoms of each participant. Participants were divided into responder and non-responder groups according to symptom improvement on the basis of a $25 \%$ reduction in the baseline PANSS score after four weeks of treatment [19].

We also recruited seventy-nine controls in good physical health (male/female $=44 / 35$ ) from the local community in Yangzhou, with an average age of $35.92 \pm 11.00$ years and mean time in education of $12.48 \pm 3.77$ years. The health status of subjects were identified by physical examination and laboratory tests. Participants who had a family history of mental illness or were diagnosed with any Axis I disorders were excluded.

All participants in this study gave informed written consent, which was approved by the Ethics Committee of Wu Tai Shan Hospital.

\subsection{NRG1ß1 assessment}

We collected venous blood samples from patients at baseline and after four weeks of medication, while healthy controls were only sampled at baseline. All venous blood samples from both patients and healthy controls were collected from the forearm vein between 07:00 and 09:00. The samples were tested by the same investigator and our research team was blind to the clinical status of this study. Peripheral blood was separated by centrifugation at $3000 \mathrm{~g}$ for $15 \mathrm{~min}$ and then stored at $-80^{\circ} \mathrm{C}$ until use. Serum NRG1 $\beta 1$ levels were tested using sandwich enzyme-linked immunosorbent assays (ELISA) according to the manufacturer's instructions (DY377; R\&D Systems, Minneapolis, MN, USA). The serum NRG1 11 levels 
were expressed in $\mathrm{ng} / \mathrm{mL}$ and the intra-assay and inter-assay variation coefficients were $<5 \%$, with 0.125 $\mathrm{ng} / \mathrm{mL}$ as the lower detection limit.

\subsection{Statistical analysis}

The Statistical Package for Social Sciences (SPSS) 19.0 was used to analyze all of the data. Chi-square tests were used to compare categorical variables. The normal distribution of the dataset was examined using the Kolmogorov-Smirnov test. Numerical variables were expressed as mean \pm standard deviation (SD). Independent sample t-tests or paired samples t-tests were performed for normality continuous variables. Analysis of covariance (ANCOVA) was used to analyze potentially confounding variables such as sex, age and years of education. Relationships between serum NRG1 $\beta 1$ levels and normality continuous variables were assessed using Pearson's correlation coefficients, while relationships with nonparametric continuous variables were evaluated using Spearman's correlation coefficients. We used stepwise regression analysis to investigate the associations between NRG1 $\beta 1$ serum levels and demographic data and clinical characteristics. Effect size determination and power calculation were carried out using the $G *$ Power3 program [20]. Results were considered significant at the $p<0.05$ level.

\section{Results}

\subsection{Demographic data}

Table 1 shows the detailed data on the demographic differences between patients with schizophrenia and healthy controls. Except for years of education, there were no significant differences in sex and age between patients with schizophrenia and healthy controls $(p>0.05)$. There were no significant correlations between NRG1 $\beta 1$ levels and sex, age and education between the two groups $(p>0.05)$. The mean dose of antipsychotic drugs (chlorpromazine equivalent) was $638.04 \pm 268.95 \mathrm{mg} /$ day. In the patient group, duration of illness, age at schizophrenia onset, and antipsychotic dose were not associated with NRG1 1 levels (all $p>0.05$ ). Table 1 also shows the significant reduction in PANSS scores and its three subscales before and after treatment (all $\mathrm{P}=0.000$ ). 
Table 1

Demographic data of patients with schizophrenia and healthy controls.

\begin{tabular}{|c|c|c|c|c|}
\hline & Controls $(n=79)$ & \multicolumn{2}{|c|}{ Schizophrenia $(n=100)$} & $p$-value \\
\hline $\operatorname{Sex}(M / F)$ & $44 / 35$ & \multicolumn{2}{|l|}{$55 / 45$} & $0.926 a$ \\
\hline Age (years) & $35.92 \pm 11.01$ & \multicolumn{2}{|l|}{$35.07 \pm 11.42$} & $0.614 b$ \\
\hline Time in education (years) & $12.48 \pm 3.77$ & \multicolumn{2}{|l|}{$10.40 \pm 3.43$} & $0.000 \mathrm{~b}$ \\
\hline Age at schizophrenia onset & & \multicolumn{2}{|l|}{$29.59 \pm 5.37$} & \\
\hline \multirow[t]{2}{*}{ Duration of illness (years) } & & \multicolumn{2}{|l|}{$10.64 \pm 7.58$} & \\
\hline & & Before treatment & After treatment & \\
\hline PANSS total score & & $77.49 \pm 6.22$ & $49.21 \pm 11.18$ & $0.000 c$ \\
\hline P subscore & & $24.34 \pm 7.56$ & $16.13 \pm 5.48$ & $0.000 c$ \\
\hline N subscore & & $20.84 \pm 7.33$ & $13.30 \pm 4.21$ & $0.000 c$ \\
\hline G subscore & & $32.31 \pm 4.42$ & $19.78 \pm 6.24$ & $0.000 c$ \\
\hline
\end{tabular}

b independent sample t-test.

c paired samples t-test.

\subsection{NRG1ß1 level}

Independent sample t-tests showed that baseline serum NRG1 $\beta 1$ levels in the patient group were significantly decreased compared with the control group $(7.58 \pm 4.03$ vs $11.87 \pm 6.69 \mathrm{ng} / \mathrm{mL}, \mathrm{F}=42.918, \mathrm{t}$ $=-5.030, P<0.000$ ) with an effect size of 0.36 and a power of 0.78 . Paired samples t-tests showed that baseline serum NRG1 11 levels were statistically lower for patients with schizophrenia compared with those after treatment $(7.58 \pm 4.03 \mathrm{vs} 10.89 \pm 6.97 \mathrm{ng} / \mathrm{mL}, \mathrm{t}=-4.341, \mathrm{P}=0.000)$ with an effect size of 0.27 and a power of 0.58 (Fig. 1). Significant differences remained when testing for covariance with sex, age and education as covariates $(P<0.000)$ In addition, no significant differences in the NRG1 $\beta 1$ levels were detected between the patient group after antipsychotic treatment and healthy controls $(t=0.951, P=$ 0.343; Fig. 1). In the patient group, there was no significant correlation between the PANSS total and its subscale scores, or NRG1 $\beta 1$ serum levels (all $P>0.05$ ).

\subsection{Relationship between NRG1ß1 and clinical efficacy in responders}

Patients with schizophrenia showing a PANSS score after treatment greater than $25 \%$ different from baseline were considered as responders. After four weeks antipsychotic drug treatment, $85 \%$ of patients 
were classified as responders, while $15 \%$ of patients were placed into the non-responder group. In the responder group, serum NRG1 $\beta 1$ levels were markedly higher after treatment $(11.32 \pm 7.00 \mathrm{ng} / \mathrm{mL})$ than before treatment $(7.50 \pm 4.21 \mathrm{ng} / \mathrm{mL})(t=4.555, P=0.000$, Fig. 2$)$, with an effect size of 0.31 and a power of 0.66 . In contrast, there was no significant difference in the level of NRG1 11 before and after treatment $(8.03 \pm 2.91 \mathrm{vs} 8.44 \pm 6.49 \mathrm{ng} / \mathrm{mL})$ in the non-responder group $(t=0.250, P=0.806$, Fig. 2$)$.

In the responder group after treatment, Pearson's correlation coefficients showed a correlation between NRG1 $\beta 1$ levels and improvements in symptoms $(r=0.223, P=0.04)$. Multiple regression analyses adjusting for potentially confounding variables showed no relationships between NRG1 $\beta 1$ levels and sex, age, time in education and age at schizophrenia onset, but confirmed a relationship between NRG1 $\beta 1$ levels and the duration of illness (beta $=-0.234, P=0.032$ ).

\section{Discussion}

The results of this study demonstrated that: 1) NRG1 $\beta 1$ levels were lower in FEDN patients with chronic schizophrenia than in healthy controls; 2 ) levels of NRG1 $\beta 1$ increased significantly after antipsychotic treatment along with improvements in psychotic symptoms; and 3) NRG1 11 levels were negatively associated with the duration of illness in the responder group. To date, few studies have examined the relationship between serum NRG1 $\beta 1$ levels and antipsychotic medication in FEDN patients with chronic schizophrenia.

This study found decreased serum levels of NRG1ß1 in FEDN patients and chronic schizophrenia, which is consistent with the results of another study of patients of Han Chinese ancestry, indicating significantly lower NRG1 levels in participants with first episode schizophrenia and chronic schizophrenia compared with healthy controls [21]. However, there are contradictory findings reported in the literature; one study reported elevated cleavage of neuregulin-1 by $\beta$-Secretase (BACE1) in the plasma of schizophrenia patients and a relationship between BACE1-cleaved-NRG1 activity and both disease severity and disease duration [22]. Moreover, Yamamori et al measured the mRNA expression levels of NRG1 in immortalized lymphocytes and found no difference in the expression of the NRG1 gene between schizophrenic patients and controls [23]. Numerous factors may account for these differences, such as demographic differences (age, sex, time in education, body mass index, smoking, or ethnicity), age at schizophrenia onset, drug treatment, duration of illness, disease severity, or methodological or test protocol differences. Different degrees of genetic variations or differences in the subtypes of schizophrenic patient may also have contributed to the discrepancies in the reported serum NRG1 levels. The decreased serum NRG1 $\beta 1$ levels found in this study accord with those observed in the brains of schizophrenics. For example, significantly reduced NRG-1 a protein levels have been found in the white matter of the prefrontal cortex [24]. Furthermore, Hashimoto et al. reported a decrease of NRG-1 type II/type I and type II/type III mRNA expression ratios in the prefrontal cortex (PFC) of schizophrenic patients [25]. Nonetheless, our evidence suggests that variation in NRG1 $\beta 1$ levels may be responsible for the pathophysiology and clinical manifestation of schizophrenia, even though the underlying mechanisms remain largely unknown. 
It was noteworthy that this study demonstrated that elevated NRG1 11 levels are associated with the early stage therapeutic effects of typical antipsychotic treatments. The detected NRG1 $1 \beta 1$ levels were markedly higher in responders after four weeks of treatment, whereas no differences were observed in nonresponders. Additionally, in a clinical study of a 2-week treatment of first-onset schizophrenic patients (who had not taken antipsychotics before) with the antipsychotics risperidone and quetiapine, the NRG1 mRNA expression of peripheral blood lymphocytes (PBLs) gradually and significantly increased after therapy, compared with the pretreatment levels [16]. Although different atypical antipsychotic drugs were administered to responders, we speculate that increased NRG1 $\beta 1$ plays an important part in achieving effective medication and improvement of psychotic symptoms through its effects on the various signaling pathways. The exact pharmacological and pathophysiological mechanisms remain obscure and warrant further investigation.

Several animal studies have shown that NRG1/ErbB4 (the NRG1 receptor) expression can be altered by treatment with drugs for chronic psychosis. Dang et al. found that haloperidol and risperidone both increase the expression of NRG1 in the brains of rats during a 4-week treatment [26]. Hahn et al. reported that NRG1-induced ErB4 activation is significantly reduced in the brains of mice treated with haloperidol compared with controls treated with vehicle alone, and that enhanced NRG1 signaling may contribute to $\mathrm{N}$-methyl-D-aspartate (NMDA) receptor hypofunction in schizophrenia [27]. In particular, one previous study found that NRG1 (both endogenous and exogenous) plays an important role in maintaining evoked GABA release in the rat prefrontal cortex (GABAergic dysfunction is implicated in schizophrenia), suggesting that NRG1 may be involved in interactions with other signaling pathways which could be useful in understanding the pathogenesis of schizophrenia and the effects of treatment [28]. However, there was no significant effect of chronic haloperidol treatment on serum Ig-NRG1 immunoreactivity in monkeys after eight weeks haloperidol treatment [29]. An in vitro study has shown that NRG1 protein expression is upregulated in human fetal brain aggregates exposed to clozapine; however, these effects were not seen in haloperidol exposed brain aggregates [30]. Taken together, these studies support a potential mode of therapeutic action for antipsychotics via NRG1-ErbB4 signaling which contributes to treatment efficacy in controlling schizophrenia symptoms.

Generally, a longer duration of illness hinders treatment response and is related to more severe negative symptoms. Earlier intervention could shorten the duration of illness and lead to a more favorable prognosis in schizophrenic patients [31]. Our analysis demonstrated that there was a negative correlation between NRG1 11 level and duration of illness in responders. The results showed that patients with longer histories of schizophrenia may have lower NRG1 1 levels, indicating that the length of illness is associated with nervous system impairments, leading to perturbed NRG1 11 synthesis, release and modulation of neuronal activity. Shorter periods of illness inflict less damage on the central nervous system and may allow for better therapeutic outcomes. Kataria et. al showed that NRG1/ErbB participates in different neurodevelopmental processes and is involved in dopaminergic neurotransmission as well as the survival of dopaminergic neurons [32]. Interestingly, we found a positive correlation between NRG1 $\beta 1$ levels and improvement in symptoms in responders. One explanation for the underlying mechanism is that the relationship between NRG1 11 levels and improvement in symptoms 
may be the result of the bodies' ability to properly establish positive feedback mechanisms to counter neuroimmune system damage. Therefore, in view of the relationship between NRG1 $\beta 1$ levels and the duration of illness and symptoms, we conjecture that NRG1 $\beta 1$ may be an indicator of therapeutic effect and its progress in schizophrenia through interactions between neurotransmitters and neurotrophic biomarkers. This potential mechanism requires further study.

There are some limitations to this study. First, although effect size was estimated prior to the study, the sample size is still small. Second, the follow-up period was relatively short. Third, different atypical antipsychotics may have different influences on serum NRG1 11 levels, and further work is needed to compare the effects of different antipsychotics on NRG1 11 levels. Fourth, our study was cross-sectional in nature and could not reveal direct causality between NRG1 11 levels and psychopathology; a longitudinal design or prospective study is needed to address this. Fifth, smoking or body mass index (BMI) may contribute to variations in NRG1 11 levels in the schizophrenia group and regulation for confounding factors is necessary to confirm our results.

In conclusion, this study demonstrated that serum NRG1 $1 \beta 1$ levels increased in FEDN patients with chronic schizophrenia after medication with atypical antipsychotic drugs alongside improvements in psychotic symptoms. These altered NRG1 $\beta 1$ levels may be associated with the progression of illness and response to treatment. This study provides evidence that NRG1 11 may be involved in the pathophysiological and potential therapeutic mechanisms of schizophrenia, although the underlying pathways still require further investigation.

\section{Declarations}

\section{Acknowledgements:}

We would like to thank Dr. Xiaowei Tang, Yaqin Zhao and Fei Ye for all of their hard work and significant contributions toward the study.

\section{References}

1. Charlson FJ, Ferrari AJ, Santomauro DF, Diminic S, Stockings E, Scott JG, McGrath JJ, Whiteford HA: Global Epidemiology and Burden of Schizophrenia: Findings From the Global Burden of Disease Study 2016. Schizophrenia bulletin 2018, 44(6):1195-1203.

2. Stepnicki P, Kondej M, Kaczor AA: Current Concepts and Treatments of Schizophrenia. Molecules 2018, 23(8).

3. Jena M, Ranjan R, Mishra BR, Mishra A, Nath S, Sahu P, Meher BR, Srinivasan A, Maiti R: Effect of lurasidone vs olanzapine on neurotrophic biomarkers in unmedicated schizophrenia: A randomized controlled trial. J Psychiatr Res 2019, 112:1-6. 
4. Ruiz de Azua S, Matute C, Stertz L, Mosquera F, Palomino A, de la Rosa I, Barbeito S, Vega P, Kapczinski F, González-Pinto A: Plasma brain-derived neurotrophic factor levels, learning capacity and cognition in patients with first episode psychosis. BMC psychiatry 2013, 13:27.

5. Agim ZS, Esendal M, Briollais L, Uyan O, Meschian M, Martinez LA, Ding Y, Basak AN, Ozcelik H: Discovery, validation and characterization of Erbb4 and Nrg1 haplotypes using data from three genomewide association studies of schizophrenia. PLoS One 2013, 8(1):e53042.

6. Fornasari BE, El Soury M, De Marchis S, Perroteau I, Geuna S, Gambarotta G: Neuregulin1 alpha activates migration of neuronal progenitors expressing ErbB4. Mol Cell Neurosci 2016, 77:87-94.

7. Mei L, Xiong WC: Neuregulin 1 in neural development, synaptic plasticity and schizophrenia. Nat Rev Neurosci 2008, 9(6):437-452.

8. Buonanno A: The neuregulin signaling pathway and schizophrenia: from genes to synapses and neural circuits. Brain Res Bull 2010, 83(3-4):122-131.

9. Lewis DA, Gonzalez-Burgos G: Pathophysiologically based treatment interventions in schizophrenia. Nat Med 2006, 12(9):1016-1022.

10. Chesworth R, Rosa-Porto R, Yao S, Karl T: Sex-specific sensitivity to methamphetamine-induced schizophrenia-relevant behaviours in neuregulin 1 type III overexpressing mice. J Psychopharmacol 2021, 35(1):50-64.

11. Pan B, Huang XF, Deng C: Antipsychotic treatment and neuregulin 1-ErbB4 signalling in schizophrenia. Prog Neuropsychopharmacol Biol Psychiatry 2011, 35(4):924-930.

12. Law AJ, Lipska BK, Weickert CS, Hyde TM, Straub RE, Hashimoto R, Harrison PJ, Kleinman JE, Weinberger DR: Neuregulin 1 transcripts are differentially expressed in schizophrenia and regulated by $\mathbf{5}^{\prime}$ SNPs associated with the disease. Proceedings of the National Academy of Sciences of the United States of America 2006, 103(17):6747-6752.

13. Parlapani E, Schmitt A, Wirths O, Bauer M, Sommer C, Rueb U, Skowronek MH, Treutlein J, Petroianu GA, Rietschel $M$ et al: Gene expression of neuregulin-1 isoforms in different brain regions of elderly schizophrenia patients. World J Biol Psychiatry 2010, 11(2 Pt 2):243-250.

14. Mata I, Perez-Iglesias R, Roiz-Santianez R, Tordesillas-Gutierrez D, Gonzalez-Mandly A, VazquezBarquero $\mathrm{JL}$, Crespo-Facorro $\mathrm{B}$ : A neuregulin 1 variant is associated with increased lateral ventricle volume in patients with firstepisode schizophrenia. Biol Psychiatry 2009, 65(6):535-540.

15. Gruber O, Falkai P, Schneider-Axmann T, Schwab SG, Wagner M, Maier W: Neuregulin-1 haplotype HAP(ICE) is associated with lower hippocampal volumes in schizophrenic patients and in non-affected family members. $J$ Psychiatr Res 2008, 43(1):1-6. 
16. Zhang HX, Zhao JP, Lv LX, Li WQ, Xu L, Ouyang X, Yuan ZQ, Huang JS: Explorative study on the expression of neuregulin-1 gene in peripheral blood of schizophrenia. Neurosci Lett 2008, 438(1):1-5.

17. Mostaid MS, Lee TT, Chana G, Sundram S, Shannon Weickert C, Pantelis C, Everall I, Bousman C: Elevated peripheral expression of neuregulin-1 (NRG1) mRNA isoforms in clozapine-treated schizophrenia patients. Transl Psychiatry 2017, 7(12):1280.

18. Kastin AJ, Akerstrom V, Pan W: Neuregulin-1-beta1 enters brain and spinal cord by receptormediated transport. J Neurochem 2004, 88(4):965-970.

19. Leucht S, Davis JM, Engel RR, Kane JM, Wagenpfeil S: Defining 'response' in antipsychotic drug trials: recommendations for the use of scale-derived cutoffs. Neuropsychopharmacology 2007, 32(9):1903-1910.

20. Faul F, Erdfelder E, Lang AG, Buchner A: G*Power 3: a flexible statistical power analysis program for the social, behavioral, and biomedical sciences. Behavior research methods 2007, 39(2):175-191.

21. Wang $R$, Wang $Y$, Hu R, Chen $X$, Song $M$, Wang $X$ : Decreased plasma levels of neureglin- 1 in drug naive patients and chronic patients with schizophrenia. Neurosci Lett 2015, 606:220-224.

22. Zhang Z, Cui J, Gao F, Li Y, Zhang G, Liu M, Yan R, Shen Y, Li R: Elevated cleavage of neuregulin-1 by beta-secretase 1 in plasma of schizophrenia patients. Prog Neuropsychopharmacol Biol Psychiatry 2019, 90:161-168.

23. Yamamori H, Hashimoto R, Verrall L, Yasuda Y, Ohi K, Fukumoto M, Umeda-Yano S, Ito A, Takeda M: Dysbindin-1 and NRG-1 gene expression in immortalized lymphocytes from patients with schizophrenia. J Hum Genet 2011, 56(7):478-483.

24. Bertram I, Bernstein HG, Lendeckel U, Bukowska A, Dobrowolny H, Keilhoff G, Kanakis D, Mawrin C, Bielau $\mathrm{H}$, Falkai $\mathrm{P}$ et al: Immunohistochemical evidence for impaired neuregulin-1 signaling in the prefrontal cortex in schizophrenia and in unipolar depression. Ann N Y Acad Sci 2007, 1096:147-156.

25. Hashimoto R, Straub RE, Weickert CS, Hyde TM, Kleinman JE, Weinberger DR: Expression analysis of neuregulin-1 in the dorsolateral prefrontal cortex in schizophrenia. Mol Psychiatry 2004, 9(3):299-307.

26. Dang R, Guo Y, Cai H, Yang R, Liang D, Lv C, Jiang P: Effects of prolonged antipsychotic administration on neuregulin-1/ErbB signaling in rat prefrontal cortex and myocardium: implications for the therapeutic action and cardiac adverse effect. The Journal of toxicological sciences 2016, 41(2):303309.

27. Hahn CG, Wang HY, Cho DS, Talbot K, Gur RE, Berrettini WH, Bakshi K, Kamins J, Borgmann-Winter $\mathrm{KE}$, Siegel SJ et al: Altered neuregulin 1-erbB4 signaling contributes to NMDA receptor hypofunction in schizophrenia. Nat Med 2006, 12(7):824-828. 
28. Woo RS, Li XM, Tao Y, Carpenter-Hyland E, Huang YZ, Weber J, Neiswender H, Dong XP, Wu J, Gassmann M et al: Neuregulin-1 enhances depolarization-induced GABA release. Neuron 2007, 54(4):599610.

29. Shibuya M, Komi E, Wang R, Kato T, Watanabe Y, Sakai M, Ozaki M, Someya T, Nawa H:

Measurement and comparison of serum neuregulin 1 immunoreactivity in control subjects and patients with schizophrenia: an influence of its genetic polymorphism. J Neural Transm (Vienna) 2010, 117(7):887-895.

30. Chana G, Lucero G, Salaria S, Lozach J, Du P, Woelk C, Everall I: Upregulation of NRG-1 and VAMP1 in human brain aggregates exposed to clozapine. Schizophr Res 2009, 113(2-3):273-276.

31. Murru A, Carpiniello B: Duration of untreated illness as a key to early intervention in schizophrenia: A review. Neurosci Lett 2018, 669:59-67.

32. Kataria $\mathrm{H}$, Alizadeh A, Karimi-Abdolrezaee S: Neuregulin-1/ErbB network: An emerging modulator of nervous system injury and repair. Prog Neurobiol 2019, 180:101643.

\section{Figures}

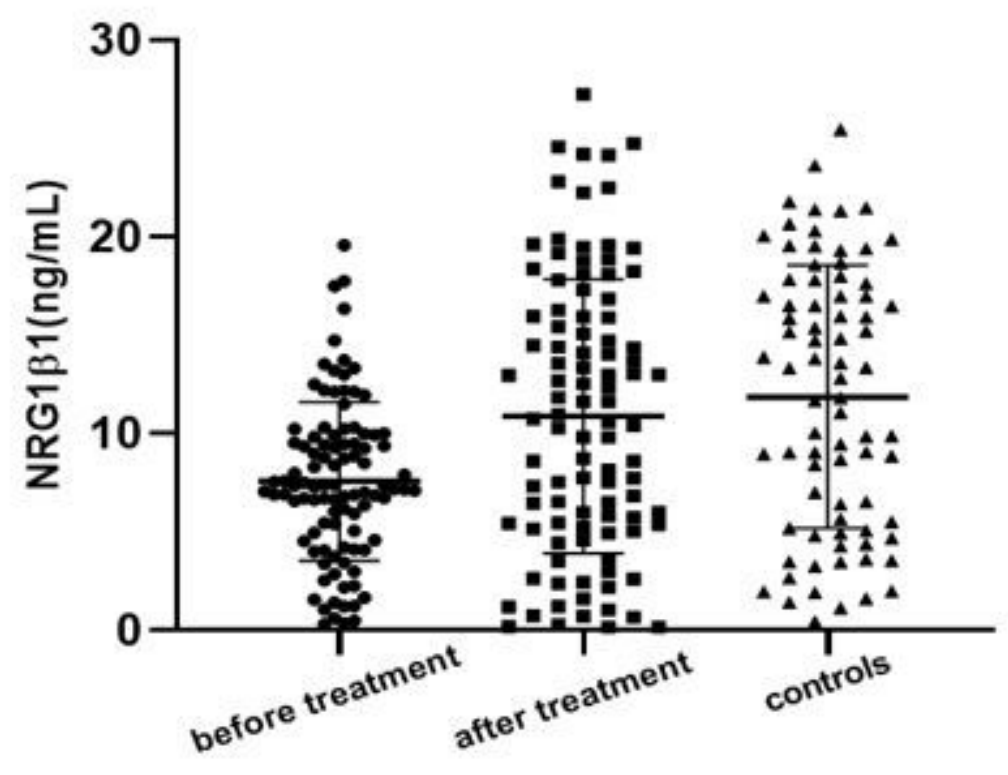

Figure 1

NRG1 11 levels in the patient and control groups before and after antipsychotic drug treatment. 


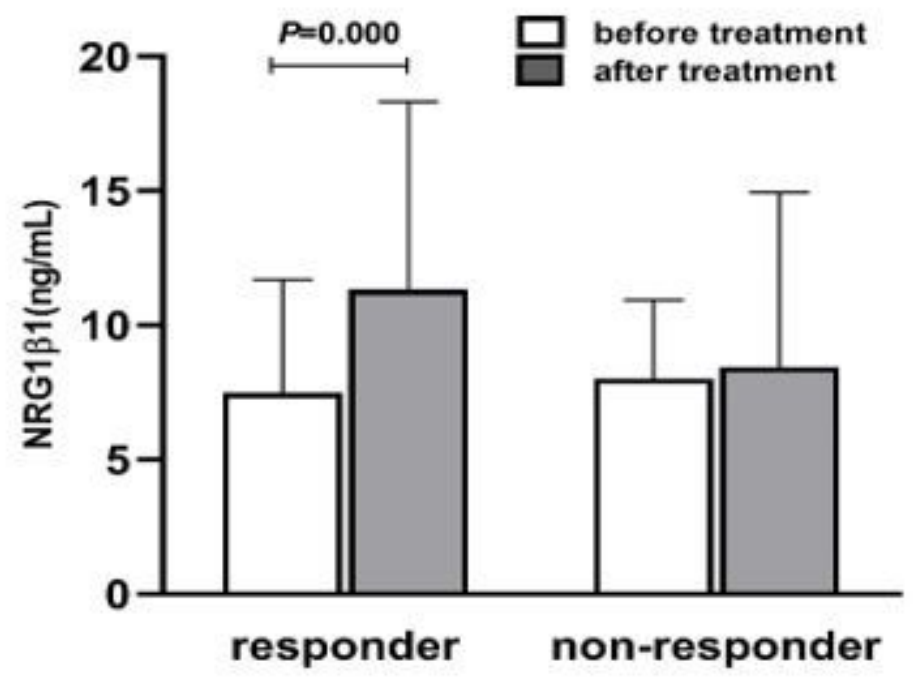

Figure 2

NRG1 $\beta 1$ levels in the responder and non-responder groups before and after antipsychotic drug treatment. 